\title{
Broca's area, sentence comprehension, and working memory: an fMRI study
}

\author{
Corianne Rogalsky, William Matchin and Gregory Hickok* \\ Center for Cognitive Neuroscience \& Department of Cognitive Sciences, University of California, Irvine, USA \\ Edited by: $\quad$ Russell A. Poldrack, University of California, USA \\ Reviewed by: Joseph T. Devlin, University College London, UK \\ Jason M. Chein, Temple University, USA
}

The role of Broca's area in sentence processing remains controversial. According to one view, Broca's area is involved in processing a subcomponent of syntactic processing. Another view holds that it contributes to sentence processing via verbal working memory. Sub-regions of Broca's area have been identified that are more active during the processing of complex (object-relative clause) sentences compared to simple (subject-relative clause) sentences. The present study aimed to determine if this complexity effect can be accounted for in terms of the articulatory rehearsal component of verbal working memory. In a behavioral experiment, subjects were asked to comprehend sentences during concurrent speech articulation which minimizes articulatory rehearsal as a resource for sentence comprehension. A finger-tapping task was used as a control concurrent task. Only the object-relative clause sentences were more difficult to comprehend during speech articulation than during the manual task, showing that articulatory rehearsal does contribute to sentence processing. A second experiment used fMRI to document the brain regions underlying this effect. Subjects judged the plausibility of sentences during speech articulation, a finger-tapping task, or without a concurrent task. In the absence of a secondary task, Broca's area (pars triangularis and pars opercularis) demonstrated an increase in activity as a function of syntactic complexity. However, during concurrent speech articulation (but not finger-tapping) this complexity effect was eliminated in the pars opercularis suggesting that this region supports sentence comprehension via its role in articulatory rehearsal. Activity in the pars triangularis was modulated by the finger-tapping task, but not the speech articulation task.

Keywords: Broca's area, fMRI, language, working memory, syntax

\section{INTRODUCTION}

The role of Broca's area in sentence processing has been debated for the last 30 years. Broca's area (defined as the posterior portions of the inferior frontal gyrus, i.e. the pars triangularis and pars opercularis) has been implicated in syntactic processing (Bradley et al., 1980; Caramazza and Zurif, 1976; Grodzinsky, 1984, 2000), but also has been proposed as a verbal working memory resource for sentence comprehension (Caplan and Waters, 1999; Just and Carpenter, 1992; Martin, 2003). The study described here examined the relationship between sentence comprehension, verbal working memory, and Broca's area.

\section{BROCA'S AREA AND SYNTACTIC PROCESSING}

Broca's area has long been implicated in sentence processing (Goodglass and Kaplan, 1972). Broca's area was first thought to support syntactic processing because Broca's aphasics were found to have agrammatic production ${ }^{1}$ (Gleason et al., 1975;

*Correspondence: Gregory Hickok, Department of Cognitive Sciences, University of California, Irvine, 3151 Social Sciences Plaza, Irvine, CA 92697, USA. e-mail: greg. hickok@uci.edu

Received: 11 August 2008; paper pending published: 28 August 2008; accepted: 24 September 2008; published online: 06 October 2008.

Citation: Front. Hum. Neurosci. (2008) 2: 14. doi: 10.3389/neuro.09.014.2008

Copyright $\odot 2008$ Rogalsky, Matchin and Hickok. This is an open-access article subject to an exclusive license agreement between the authors and the Frontiers Research Foundation, which permits unrestricted use, distribution, and reproduction in any medium, provided the original authors and source are credited.
Goodglass, 1968, 1976; Goodglass and Berko, 1960; Kean, 1977). Such patients typically produce syntactically simple sentences that lack function words and inflections (Kean, 1995), suggesting that they are lacking the ability to form syntactic structures. Broca's aphasics also have been found to have difficulty comprehending syntactically demanding utterances such as semantically reversible sentences that contained non-canonical word order ("It was the squirrel that the raccoon chased") (Bradley et al., 1980; Caramazza and Zurif, 1976). Such sentences place a heavy burden on syntactic mechanisms because meaning cannot be inferred from lexical-semantic information alone (c.f., "It was the nut that the squirrel ate"), nor can typical Englishlanguage subject-verb-object word order patterns provide clues to the subject and object of the action (c.f., "It was the ${ }^{1}$ raccoon that chased the squirrel"). These deficits in comprehending syntactically complex sentences, along with agrammatic production deficits, suggested an overall syntactic deficit in Broca's aphasia, and thus linked syntax to Broca's area (Bradley et al., 1980; Caramazza and Zurif, 1976).

Despite early evidence implicating Broca's area in syntactic processing, subsequent studies questioned this view. For example, Broca's aphasics were found to be able to make

${ }^{1}$ Although Broca's area is often damaged in Broca's aphasia, damage to this region is neither sufficient (Mohr, 1976; Mohr et al., 1978) nor necessary (Dronkers et al., 1992) to cause Broca's aphasia. The neural basis of the comprehension pattern often found in Broca's aphasia is less than clear (Dronkers et al., 2004). This complication does not negatively affect the arguments made below, however. 
grammaticality judgments of sentences, even if they are unable to comprehend these same sentences (Linebarger et al., 1983; Wulfeck, 1988), suggesting that these patients have significant syntactic knowledge available to them (Linebarger, 1990; Linebarger et al., 1983; Martin, 2003). And while some of the earliest functional imaging studies of sentence processing implicated Broca's area, particularly in the comprehension of complex structures (Caplan et al., 1998, 1999; Dapretto and Bookheimer, 1999; Just et al., 1996; Stromswold et al., 1996), other studies found a lack of correspondence between sentence processing and activity in Broca's area. For example, Mazoyer et al. (1993) found that activity in Broca's area does not track with the presence or absence of syntactic structure, responding to meaningful sentences, but also to unstructured word lists, and not to structured sentences that are semantically odd. In contrast, another region, the anterior temporal lobe, does appear to track with the presence of syntactic structure, and consequently has emerged as a candidate for supporting syntactic and/or compositional semantic processing (Humphries et al., 2001, 2005; Mazoyer et al., 1993; Rogalsky and Hickok, 2008; Vandenberge et al., 2002). Thus, the role of Broca's area in sentence processing needs to be reevaluated.

There are two main theories regarding the contributions of Broca's area to sentence processing. One is that it is involved in a subcomponent of syntactic processing (e.g., processing longdistance dependencies) (Grodzinsky, 2000). The other is that Broca's area is involved in working memory processes that support sentence comprehension in high-load conditions (Caplan and Waters, 1999; Just and Carpenter, 1992; Martin, 2003). The current study directly addresses this latter hypothesis.

\section{WORKING MEMORY AND SENTENCE PROCESSING}

There are a variety of conceptualizations of "working memory" that have been investigated in connection with sentence comprehension (Caplan and Waters, 1999; Just and Carpenter, 1992; Martin, 2003). We will focus here on one of them, namely that the comprehension of complex sentences requires verbal information to be stored in something like Baddeley's phonological loop (Baddeley, 1986; Baddeley and Hitch, 1974). There is evidence both for and against this link between the phonological loop and sentence comprehension.

Some of the evidence supporting the involvement of the phonological loop in sentence comprehension has emerged from reading comprehension and short-term memory studies involving concurrent task paradigms (e.g. Baddeley, 1981; Baddeley et al., 1975; Besner and Davelaar, 1982; Coltheart, 1993). For example, Baddeley (1981) asked subjects to detect word order errors in visually-presented sentences while performing one of two tasks concurrently. In one condition, subjects performed the error detection task during concurrent continual articulation of the word "the." By engaging the phonological loop in the rehearsal of "the", Baddeley hypothesized that the loop's contribution to reading would be suppressed, and thus the extent of its involvement in reading could be determined. In the other concurrent task condition, subjects tapped one of their fingers, paced by a metronome. Performance in the word error detection task was significantly worse during concurrent articulation than during concurrent tapping, suggesting that the phonological loop contributes to reading processes.

Several other reading comprehension studies, using various measures of text comprehension and various concurrent articulation and tapping tasks, have found a similar impairment in reading comprehension during concurrent articulation compared to concurrent tapping (De Beni et al., 2005; Slowiaczek and Clifton, 1980; Takahashi, 2007). It is important to note that the difference in performance on the reading comprehension tasks during concurrent articulation and during tapping is not likely due to differences in the general difficulty of or attention required in the two concurrent tasks. Although comprehension performance was lower during articulation that during tapping, some of these same studies measured reaction time and reading speed: there were no significant differences in reaction times or in reading speeds between the two concurrent task conditions (Baddeley, 1981; De Beni et al., 2005). In addition, irrelevant sounds and speech presented during reading comprehension tasks do not impair reading comprehension as concurrent articulation does (Boyle and Coltheart, 1996). It is unclear, however, how comprehension as measured by reading may differ from auditory comprehension, which is the focus of the present study.

Studies of auditory comprehension also suggest that the phonological loop is involved in sentence comprehension. Imaging studies of verbal working memory have regularly implicated Broca's area as part of the phonological loop, particularly in the articulatory rehearsal component (Smith et al., 1998; Zatorre et al., 1992). Additional studies have implicated working memory resources, (but not necessarily a phonological loop) in sentence processing: Broca's aphasics have been found to have reduced digit and sentence working memory spans, and their sentence comprehension in high load situations has been correlated with verbal working memory capacity more broadly defined (Daneman and Carpenter, 1980; De Renzi and Nichelli, 1975; Just and Carpenter, 1992; Swinney and Taylor, 1971).

Although there is some evidence implicating the phonological loop in sentence comprehension, lesion data indicate that working memory span and sentence comprehension deficits are dissociable (Caplan and Waters, 1999). For example, some patients demonstrate normal comprehension of complex sentences, despite significantly restricted working memory capacities (Caplan and Waters, 1999; Hanten and Martin, 2000; Waters et al., 1991). This dissociation suggests that regions involved in the phonological loop may not be necessary for sentence comprehension (Caplan and Hildebrandt, 1988; Caplan and Waters, 1999).

A PET study by Caplan et al. (2000) provides further suggestive evidence that the phonological loop is not the basis for Broca's area's involvement in sentence comprehension. Their study compared neural activity during the comprehension of written sentences of varying syntactic complexity during articulatory suppression (repeatedly articulating a word or sequence of syllables). As mentioned above, articulatory suppression interferes with short-term memory, presumably by preventing the use of the articulatory rehearsal component of the phonological loop (Baddeley et al., 1975). In a functional imaging context, one can use articulatory suppression to assess the extent to which sentence complexity effects in Broca's area reflect articulatory rehearsal: if the complex sentences yield greater activation in Broca's area than more simple sentences even during articulatory suppression, then the residual activation can be attributed to mechanisms other than articulatory rehearsal. Caplan et al. (2000) report precisely this pattern: during articulatory suppression, they found an increase in activity in a portion of Broca's area (in the pars triangularis) for processing syntactically 
complex sentences, compared to canonical sentence forms. The authors suggest that this difference in activity "is not due to subvocal rehearsal of those structures, but rather results from processing syntactic forms themselves".

The design of Caplan et al.'s experiment limits interpretation of their findings, however. First, unlike most studies of syntactic comprehension in aphasia, they used written materials in a selfpaced format. Presenting the entire sentence and allowing the duration of presentation to be paced by the subject may have allowed subjects to re-read and/or spend more time reading the more complex sentences. It is unclear whether these findings will generalize to auditory comprehension. Second, they did not separately assess the brain activation foci associated with sentence comprehension versus articulatory rehearsal. This leaves open the possibility that articulatory suppression did saturate a portion of the normal sentence-related activation in Broca's area, suggesting some role for the working memory in sentence comprehension. Finally, they did not include a control concurrent task, such as finger tapping. The use of such a task could have revealed a contribution of working memory to the "sentence effect" in Broca's area if, for example, a larger extent of Broca's area was activated for sentences during the manual task compared with during the speech articulation task.

The aim of the current study was to address the role of phonological working memory in sentence comprehension by expanding upon Caplan et al.'s study. Our hypothesis is that at least a part of the contribution of Broca's area to sentence comprehension stems from its involvement in verbal working memory. Specifically, we hypothesized that comprehension of high load sentences is supported, in part, by recruitment of verbal working memory resources including the articulatory rehearsal mechanism. This hypothesis predicts that sentence comprehension performance on high load sentences should decline during articulatory suppression (relative to low load sentences and relative to a control task), and that at least a portion of the activation in Broca's area during high load sentences should be equally activated during articulatory suppression such that the sentence complexity effect typically found in Broca's region (high $>$ low complexity) should be eliminated. We tested these predictions in the following two experiments.

\section{EXPERIMENT 1: INVESTIGATION OF THE EFFECTS OF CONCURRENT ARTICULATION AND MANUAL SEQUENCING TASK ON SENTENCE COMPREHENSION \\ METHODS}

If in fact a phonological loop is necessary for sentence comprehension, particularly for the comprehension of syntactically complex sentences, we should be able to detect behaviorally a decrease in comprehension performance as a function of sentence complexity during concurrent speech articulation. Thus, 55 native English-speaking right-handed subjects were administered a modified SOAP (Subject Object Active Passive) sentence comprehension test (Love and Oster, 2002) during both concurrent articulation and a finger-tapping sequence task, respectively. The finger-tapping sequence task condition provided a control for the general effect of performing a secondary task during auditory comprehension.

The SOAP test consists of 40 semantically reversible sentences (e.g.: The man pushes the boy. The boy pushes the man) (10 active, 10 passive, 10 subject-relative clause (SR), and 10 object-relative clause (OR) sentences). Sentences were spoken and recorded by a female speaker using Audacity sound-editing software. The sentences were presented via headphones. Subjects were asked to match the sentence with one of three pictures. The picture arrays contained one correct picture, a picture of actors performing the reverse thematic roles, and an unrelated picture (Figure 1). The picture display was presented on a computer monitor; the auditory and visual stimuli were delivered via Cogent 2000 software (FIL, 2000) and Matlab (Mathworks, Inc.). The sentences and picture displays were presented simultaneously. Subjects were instructed to respond by pointing to the picture that matched the sentence immediately after the completion of the sentence presentation. During the training periods (described below), subjects were instructed that sentences could not be re-presented; they also were coached to
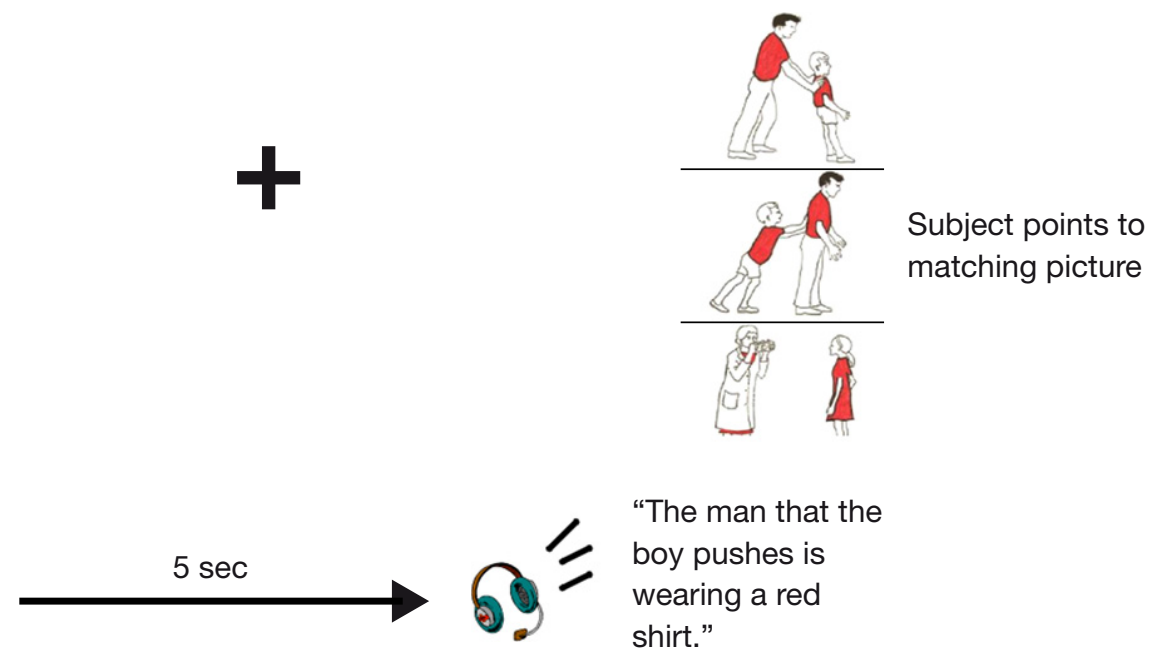

"ba da ga da...ba da ga da" or finger tapping sequence

Figure 1 | Schematic of behavioral task paradigm with SOAP stimuli (Love and 0ster, 2002). Note that the concurrent task condition was employed during both the initial perception and processing of the sentence, as well as during the post-processing matching task. 
respond immediately after the sentence presentation. Responses were recorded by the experimenter.

In the sequential finger-tapping condition, subjects placed their right hand on a table adjacent to the computer monitor. They were instructed to continuously tap their fingers on the

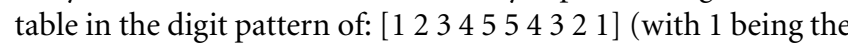
thumb, 2 the index finger, etc.). This task was intended to serve as a baseline to control for the effects of performing a secondary task during sentence comprehension.

The (speech) articulatory suppression condition involved whispering (to avoid auditory-input interference) a sequence of consonant-vowel combinations. Specifically, subjects continuously whispered the CV sequence "ba da ga da." Subjects were monitored closely in both task conditions to ensure that they were continuing the secondary task throughout the auditory presentation of the sentence, and during the picture-matching process.

Each subject completed the SOAP comprehension test for each secondary task condition. The order of the task conditions was counterbalanced across subjects, and the sentences were presented in a randomized order to each subject. Responses were coded by type of error (correct, semantically reversed error, unrelated error) and sentence type.

Subjects were trained on the corresponding concurrent task immediately prior to each condition. Training was considered to be complete when the subject was able to perform the secondary task correctly, while successfully completing five consecutive sentence-picture matching trials containing simple, active sentences.

\section{RESULTS}

A $2 \times 4$ repeated measures ANOVA was calculated to compare the number of comprehension errors during articulation and during the finger-tapping sequence task for the four sentence types. (All but two errors (i.e. $99.5 \%$ of errors) made by all subjects involved selecting pictures with thematic roles reversed. All errors were included in the subsequent analyses.) Main effects for both secondary task condition $(F(1,54)=11.73$, $p<0.0001)$ and sentence type $(F(3,54)=69.38, p<0.0005)$ were found, but an interaction was also present, $F(3,52)=8.45$, $p<0.0001$. Paired sample $t$-tests revealed that the interaction reflects a significant difference between articulatory suppression $(M=2.96)$ and the finger-tapping sequence task $(M=1.95)$ during the comprehension of the most complex sentence type, object-relative clause sentences, $t(54)=3.96, p<0.0001$. No significant differences between suppression types were found in the active, passive, and subject-relative clause sentences (Figure 2). A significant difference between the object-relative clause sentences and each of the other sentence types, within each task condition (Table 1), also contributed to the interaction effect noted above.

\section{DISCUSSION}

Experiment 1 investigated the effects of suppressing articulatory rehearsal during sentence comprehension. We found that concurrent articulation significantly reduces the ability to comprehend object-relative clause sentences compared to comprehension during a manual sequencing task and compared to all other sentence types studied here.

Our finding that object-relative clause sentences were more difficult to comprehend than all the other sentence types, during both concurrent tasks, mirrors previous studies that indicate that object-relative sentences are more difficult for subjects to comprehend than subject-relative, active, and passive sentences even

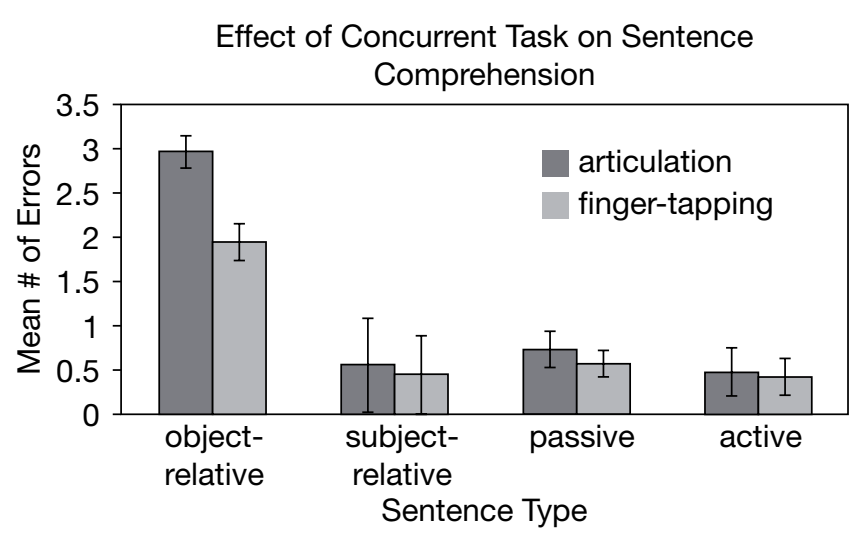

Figure 2 | Mean comprehension performance as a function of sentence type and concurrent task. Note that during each concurrent task, 10 of each sentence type were presented. Error bars represent $95 \%$ confidence intervals.

Table 1 | Summary of paired sample $\boldsymbol{t}$-tests comparing object-relative sentence comprehension with that of the other three sentence types during articulation and finger-tapping task, respectively.

\begin{tabular}{llll}
\hline Concurrent Task & Comparison & $\boldsymbol{t}$-Value $(\mathbf{d f}=\mathbf{5 4 )}$ & $\boldsymbol{p}$-Value \\
\hline Articulation & OR vs. SR & 8.98 & $<0.0001$ \\
& OR vs. passive & 9.08 & $<0.0001$ \\
& OR vs. active & 9.56 & $<0.0001$ \\
Finger-Tapping & OR vs. SR & 6.24 & $<0.0001$ \\
& OR vs. passive & 5.76 & $<0.0001$ \\
& OR vs. active & 6.51 & $<0.0001$ \\
\hline
\end{tabular}

A Bonferroni correction for multiple comparisons was implemented, controlling $\alpha_{t w}=0.05\left(\alpha_{p c}=0.0167\right)$. All comparisons were significant.

without a secondary task (Love and Oster, 2002). Lesion evidence suggests that the additional resources engaged in processing object-relative clause sentences are supported by regions including Broca's area: some patients with inferior frontal damage have more difficulty comprehending semantically reversible objectrelative clause sentences than semantically reversible subjectrelative clause sentences or active-voice sentences (Bradley et al., 1980; Caramazza and Zurif, 1976). The lack of an interference effect for passive voice sentences in our study is consistent with lesion data suggesting that performance on passive sentences is often better than that for object relative clauses sentences, despite the fact that both involve non-canonical word order (Berndt et al., 1996).

The fact that object-relative clause sentences were the only sentence type for which concurrent articulation decreased comprehension more than the manual sequencing task supports the hypothesis that verbal working memory, and the articulatory rehearsal component in particular, can help support the processing of high load sentences. Also, this result provides indirect evidence that Broca's area is contributing to sentence processing via articulatory rehearsal as previous studies have found that portions of Broca's area support both speech articulation (including during articulatory rehearsal) (Buchsbaum et al., 2001; Hickok et al., 2003; Jonides et al., 1997; Postle et al., 2001; Smith and Jonides, 1997; Smith et al., 1998) and the processing of high-load sentences relative to lower-load sentences (Caplan et al., 2008; Fiebach et al., 2005; Stromswold et al., 1996). 
The goal of experiment 2 was to determine the neural basis of these effects. Specifically, to determine to what extent the difference in Broca's area activity during the comprehension of sentences with object-relative clauses and that during subjectrelative clause sentences can be accounted for by articulatory rehearsal.

\section{EXPERIMENT 2: fMRI INVESTIGATION OF THE CONTRIBUTIONS OF BROCA'S AREA TO SENTENCE PROCESSING \\ METHODS \\ Participants}

Fifteen right-handed native English-speaking subjects participated in this fMRI experiment (nine female, age range 18-23 years, mean $=20.2$ ). All participants were free of neurological disease (self report) and gave informed consent under a protocol approved by the Institutional Review Board of the University of California, Irvine.

\section{Experiment design}

We conducted a $2 \times 3$ fMRI experiment. Expanding upon Caplan et al.'s previous studies, subjects were asked to determine the plausibility of subject-relative and object-relative sentences (i) in the absence of a secondary task, (ii) during concurrent speech articulation, and (iii) during performance of a fingertapping sequence task. The plausibility judgments involved detecting sentences that were semantically implausible (see the next section below for a description of the sentences). Responses were made via a button press. The concurrent tasks were the same as those used in the behavioral Experiment 1 described above. Measures were taken to minimize arm movement during finger-tapping (which was completed by pressing the buttons of a button box so that they could be monitored and recorded), and the articulations of the CV sequence were "low-amplitude" whispering: subjects were asked to minimize their mouth movements during articulation.

Subjects participated in 95-min scanning runs. Each run consisted of 10 trials. Each trial consisted of a 20-s task block (in which subjects were cued to articulate, perform the fingertapping sequence, or do nothing) followed by a 10 -s rest period. There were 30 trials for each task condition (15 included the presentation of object-relative sentences, and 15 included the presentation of subject-relative sentences).

Subjects were cued to begin the speech articulation task by the visual presentation of a line-drawing of a mouth, which flickered at $2 \mathrm{~Hz}$ to pace the subjects' rehearsal (thus two CVs per second). Likewise, subjects were cued to begin the fingertapping sequence by a line drawing of a hand, which also flickered at $2 \mathrm{~Hz}$ to pace the subjects' tapping (thus two finger taps per second). The corresponding picture continued to flicker during the presentation of the sentence, while the subject was making the plausibility judgment, and then for the remainder of the 20-s trial. During trials that did not include a concurrent task, a fixation cross was presented. The time between beginning the concurrent task and the presentation of the sentence was randomized within each 20 -s task block, with the restriction that the onset of a sentence was never presented earlier than $5 \mathrm{~s}$ into the task block, and no later than $5 \mathrm{~s}$ before the end of the block. Trial order was randomized for each subject.

Immediately prior to scanning, subjects were trained on both concurrent tasks and the semantic plausibility judgments separately, and then participated in one "simulated" run, which was similar to the runs that would be conducted during scanning.

\section{Sentence stimuli}

Sentences were recorded by a male speaker and edited using Audacity sound-editing software. Sentences were presented to the subjects binaurally during scanning via Resonance Technology Inc. MR-compatible digital audio system and headphones (www. mrivideo.com). Sentences were presented at a comfortable (and audible during scanning) listening level.

Ninety sentences were presented (30 in each task condition), half contained an object-relative clause, and half contained a subject-relative clause. The average duration of sentences was $3.1 \mathrm{~s}$ (range $=2.8-3.4 \mathrm{~s}$ ). Sentences were generated in pairs, with each pair containing an object-relative sentence and a subjectrelative sentence that were matched in word length and agent/ object relationship, for example:

Object Relative: The money that the robber stole was in the bank vault.

Subject Relative: The robber that stole the money was in the bank vault.

Six object-relative and six subject-relative clause sentences were modified to be semantically implausible. These sentences were rendered implausible due to thematic role incompatibility, for example:

Object Relative: \#The robber that the money stole was in the bank vault.

Subject Relative: \#The money that stole the robber was in the bank vault.

These semantically implausible sentences, distributed evenly throughout the trials of the three task conditions, were used to ensure that subjects were staying on task. Data from implausible sentences were not included in the fMRI analysis.

\section{fMRI data acquisition \& processing}

Scanning was conducted at the $3 \mathrm{~T}$ MRI scanner at the UCI Research Imaging Center. A high-resolution anatomical image was acquired, in the axial plane, with a $3 \mathrm{D}$ SPGR pulse sequence for each subject: $\mathrm{FOV}=250 \mathrm{~mm}, \mathrm{TR}=13 \mathrm{~ms}$, flip angle $=20^{\circ}$, voxel size $=1 \mathrm{~mm} \times 1 \mathrm{~mm} \times 1 \mathrm{~mm}$. Whole-brain functional MRI data was collected using echo-planar imaging, in the axial plane, using interleaved slice acquisition: SENSE factor $=1.5$, FOV $=250 \mathrm{~mm}, \mathrm{TR}=2 \mathrm{~s}, \mathrm{TE}=40 \mathrm{~ms}$, flip angle $=90^{\circ}$, voxel size $=1.95 \mathrm{~mm} \times 1.95 \mathrm{~mm} \times 5 \mathrm{~mm}$ (i.e. $5 \mathrm{~mm}$ slice thickness, no gap between slices). Nine functional scanning runs were completed for each subject, 150 volumes collected per run. MRIcro (Rorden and Brett, 2000) was used to reconstruct the highresolution structural image, and an in-house Matlab program was used to reconstruct the echo-planar images. Functional volumes were aligned to the sixth volume in the series using a 6-parameter rigid-body model to correct for subject motion (Cox and Jesmanowicz, 1999). Each volume then was spatially filtered (FWHM $=8 \mathrm{~mm}$ ) to better accommodate group analysis.

\section{fMRI data analysis}

The software package Analysis of Functional NeuroImages (AFNI) (http://afni.nimh.nih.gov/afni) was used to perform a multiple regression analysis to examine the response properties of sentence processing regions, including Broca's area, as a function of syntactic complexity and concurrent task. Regressors for each sentence type during each concurrent task (as well as 
for each sentence type in the null task trials) were constructed, and convolved with a standard hemodynamic response function to create predictor variables for analysis (Cox and Hyde, 1997). In addition, regressors for the periods before and after sentence presentation (during which tapping, articulation, or no task were being performed) were included to be able to identify regions recruited by the "concurrent" task conditions, in the absence of sentence presentation. An F-statistic was calculated for each voxel, and activation maps were created for each subject to identify regions that were more active while listening to each sentence type during each task condition compared to baseline scanner noise. The statistical maps for each subject were transformed into standardized space (Talairach and Tournoux, 1988) and resampled into $2 \times 2 \times 2 \mathrm{~mm}$ voxels. This transformation was performed using AFNI's "adwarp" program to transform the functional maps to be aligned to each subject's anatomical MRI dataset that was in standardized space: the anatomical dataset for each subject was transformed into Talairach space by manually identifying the AC-PC plane and anatomical boundaries in each subject, and then scaling each brain to the TalairachTourneaux atlas brain in AFNI.

As reviewed in the introduction, previous studies have found (i) that portions of Broca's area are more active during the presentation of syntactically complex sentences than simple sentences, and (ii) a portion of Broca's area exhibits this complexity effect during articulatory suppression. Our analysis strategy, therefore, was to identify regions modulated by syntactic complexity in the absence of a secondary task, and then compare these regions with those modulated by complexity during the secondary tasks. To this end, voxel-wise repeated-measures $t$-tests (using AFNI's " $3 \mathrm{~d} t$ test") were performed to identify voxels more active during the presentation of plausible object-relative clause sentences than during the presentation of plausible subject-relative clause sentences during each of the three concurrent task conditions across subjects.

\section{fMRI RESULTS}

\section{Behavioral results during scanning}

Our goal in this fMRI experiment was to examine the neural response during the comprehension of normal (i.e., plausible) sentences under different secondary task conditions. For this reason, we included only a small number of semantically implausible sentences to which subjects were asked to respond. These "catch trials" were used to ensure that subjects remained attentive and were comprehending the sentences during scanning. Thus, the overt behavioral task in this fMRI study was not designed to provide statistically robust behavioral data. Nonetheless, it is possible to examine the behavior responses in the scanner to determine whether a similar pattern of results holds in the fMRI study compared to the purely behavioral Experiment 1 .

In the fMRI study, accurate performance on the semantic plausibility task required subjects to press a button if they heard an implausible sentence and to withhold from pressing any button (i.e., make no response) if they heard a plausible sentence. A subject's overall error rate on this task could be influenced by response bias. Thus, performance on the semantic plausibility task was determined by using signal detection methods to estimate each subject's ability to discriminate targets (semantically implausible sentences) from non-targets (plausible sentences), corrected for response bias. Specifically, using each subject's proportion of hits (pressing a button after the presentation of an implausible sentence) and false alarms (incorrectly pressing a button after the presentation of a plausible sentence) we calculated $d^{\prime}$ statistics separately for each sentence type during each task condition. We then calculated corresponding $a^{\prime}$ statistics, which provide an estimate of proportion correct corrected for response bias (Grier, 1971). Because $a^{\prime}$ values are more readily interpretable, the analyses reported here primarily use these $a^{\prime}$ values.

Behavioral results in the scanner were consistent with behavioral results from Experiment 1. Sentence comprehension performance in the absence of a secondary task was quite good ( $>90 \%$ ) and similar for OR and SR sentences. As predicted by the behavioral study reported above, the largest difference between plausibility judgment performance of object-relative clause and subject-relative clause sentences was seen during concurrent speech articulation (Figure 3 ). Paired-sample one-tailed $t$-tests were calculated for the difference in performance (as represented by mean $a^{\prime}$ values) between sentence types during each task condition. The difference of judgment performance between the two sentence types during concurrent articulation was significant: $t(14)=2.31, p=0.03$. Differences between judgment performance for the two sentence types during the finger-tapping sequence, as well as in the absence of a concurrent task were not significant, although there was a trend for OR sentences to lead to more errors during the finger tapping task than SR sentences, as expected from Experiment 1 (Table 2).

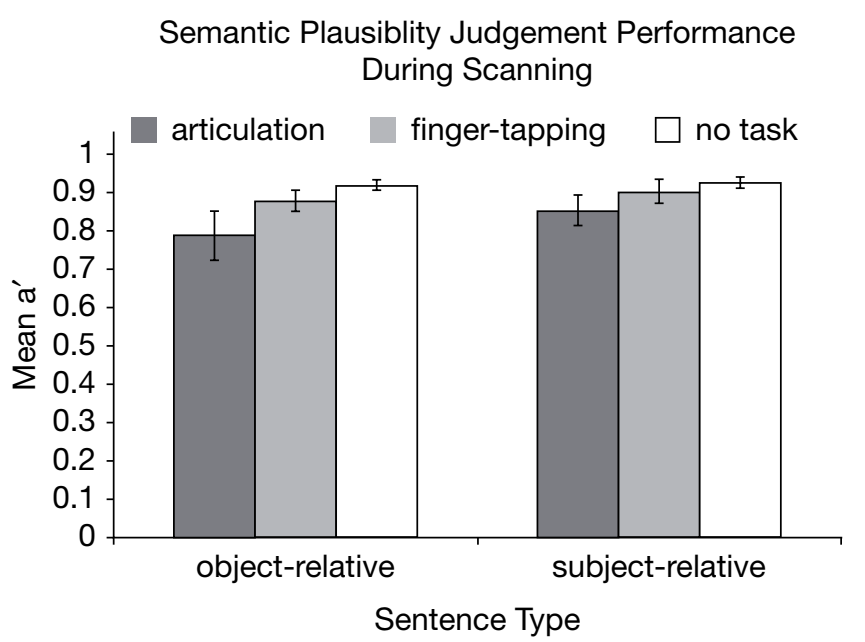

Figure 3 | Plausibility judgment performance for each sentence type in each concurrent task condition. Mean a' values across subjects for each sentence type in each task condition are depicted. Error bars represent 95\% confidence intervals.

Table 2 | Summary of paired sample $\boldsymbol{t}$-tests comparing plausibility judgment performance ( $a^{\prime}$ values averaged across subjects) for object-relative sentences and for subject-relative sentences during each concurrent task condition, as well as pair-wise comparisons for performance on each sentence type across concurrent task conditions.

\begin{tabular}{llll}
\hline Concurrent Task & OR mean a' & SR mean $\mathbf{a}^{\prime}$ & \multicolumn{1}{c}{$\boldsymbol{t} / \mathbf{p}$} \\
\hline Articulation & 0.787 & 0.854 & $2.31 / 0.03$ \\
Finger-Tapping & 0.879 & 0.904 & $1.79 / 0.09$ \\
No Concurrent Task & 0.919 & 0.925 & $1.45 / 0.16$ \\
\hline Sentence Type & Artic vs. None & Artic vs. Tap & \multirow{2}{*}{ Tap vs None } \\
\hline Object-Relative & $4.40 / 0.0003$ & $2.53 / 0.012$ & $3.09 / 0.004$ \\
Subject-Relative & $3.79 / 0.001$ & $3.23 / 0.003$ & $1.35 / 0.10$
\end{tabular}




\section{fMRI results}

We expected that the two secondary tasks themselves, speech articulation and finger tapping, would activate at least partially distinct networks given that they had differential effects on sentence comprehension in Experiment 1. Although this was not a focus of our study, for descriptive purposes, Figure 4 presents activation maps associated with speech articulation alone, finger tapping alone, and regions of overlap (conjunction) between these two tasks. This analysis indeed indicated that the two secondary tasks activated non-identical networks.

Voxel-wise paired sample $t$-tests identified regions that were more active during the plausible object-relative clause sentences than the plausible subject-relative clause sentences across subjects during (i) no secondary task, (ii) concurrent speech articulation, and (iii) concurrently performing the finger-tapping sequence task. Plausible sentences were defined as sentences that did not contain a thematic role compatibility error and were identified as plausible by the subject. Only responses to plausible sentences thus defined were analyzed; all results reported below are from this set of plausible sentences.

In the absence of a secondary task, two distinct voxel clusters in Broca's area responded more during the perception of objectrelative sentences than subject-relative sentences $(p<0.005)$ : a portion of the pars triangularis (-41 3814$)$, and a portion of the pars triangularis (-42 13 23) (Figure 5A and Table 3). Additional clusters (setting a minimum cluster threshold at
5 contiguous voxels) demonstrating this object-relative versus subject-relative sentence preference were found in the right inferior frontal gyrus (57 16 20), as well as in the left middle frontal gyrus, left pre-central gyrus, and left superior temporal gyrus (Table 3). A conjunction map of voxels responding more to the object-relative sentences than to the subject-relative sentences $(p<0.005)$ with no secondary task and the voxels active during articulation alone $(p<0.005)$ indicates that the left pars opercularis region demonstrating the complexity effect (in the absence of a secondary task), is also involved in speech articulation (Figure 6).

Many of the areas demonstrating a difference in activation between the object-relative and subject-relative sentences in the no-secondary-task condition did not exhibit this difference during concurrent articulation: only one sub-region of Broca's area, in the pars triangularis (-41339), responded more during the object-relative clause sentences than during the subject-relative clause sentences $(p<0.005)$ during concurrent articulation (Figure 5B and Table 3 ). It is notable that the pars triangularis region's preferential response to object-relative clause versus subject-relative clause sentences without a secondary task was not present during concurrent articulation even at a more liberal threshold $(p=0.10)$ (Table 4$)$. A sub-region in the right inferior frontal gyrus also responded more to the object-relative clause than subject-relative clause sentences during articulation (pars orbitalis, $3727-9$ ), as well as clusters in the right superior

\section{Regions Active During Tasks Alone (i.e. Without Sentence Presentation)}

Left Hemisphere

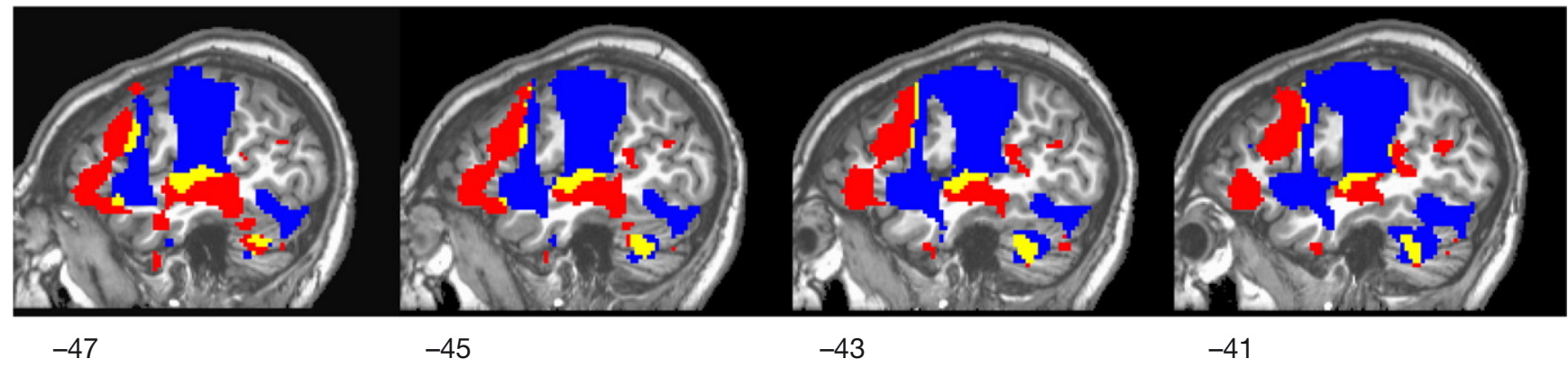

Right Hemisphere

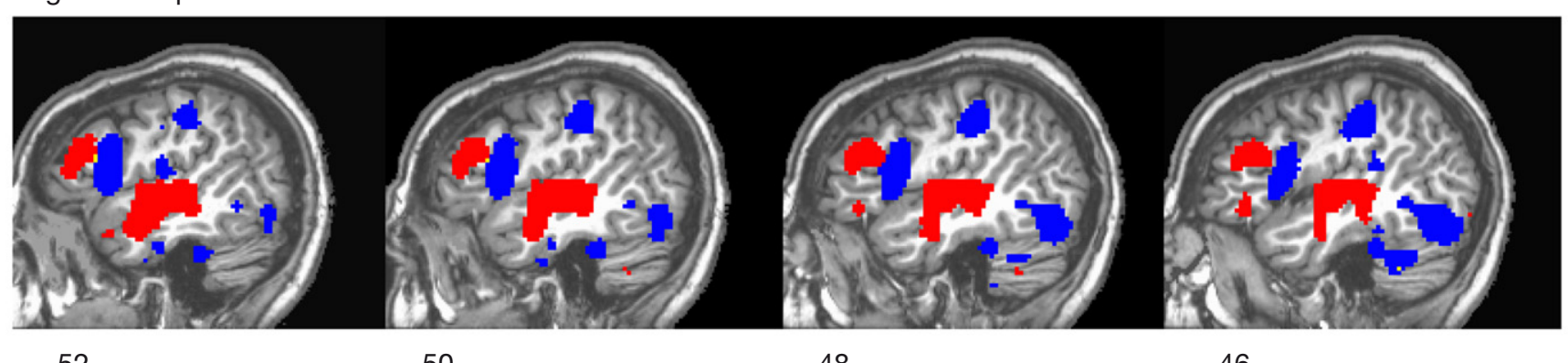

52

during articulation task

during both task during finger-tapping task

Figure 4 | Regions active across subjects during the performance of both tasks and each task, respectively, in the absence of sentence presentation, compared to rest $(p<0.005)$. 


\section{Object-Relative Clause Sentences > Subject-Relative Clause Sentences}

A No Secondary Task:
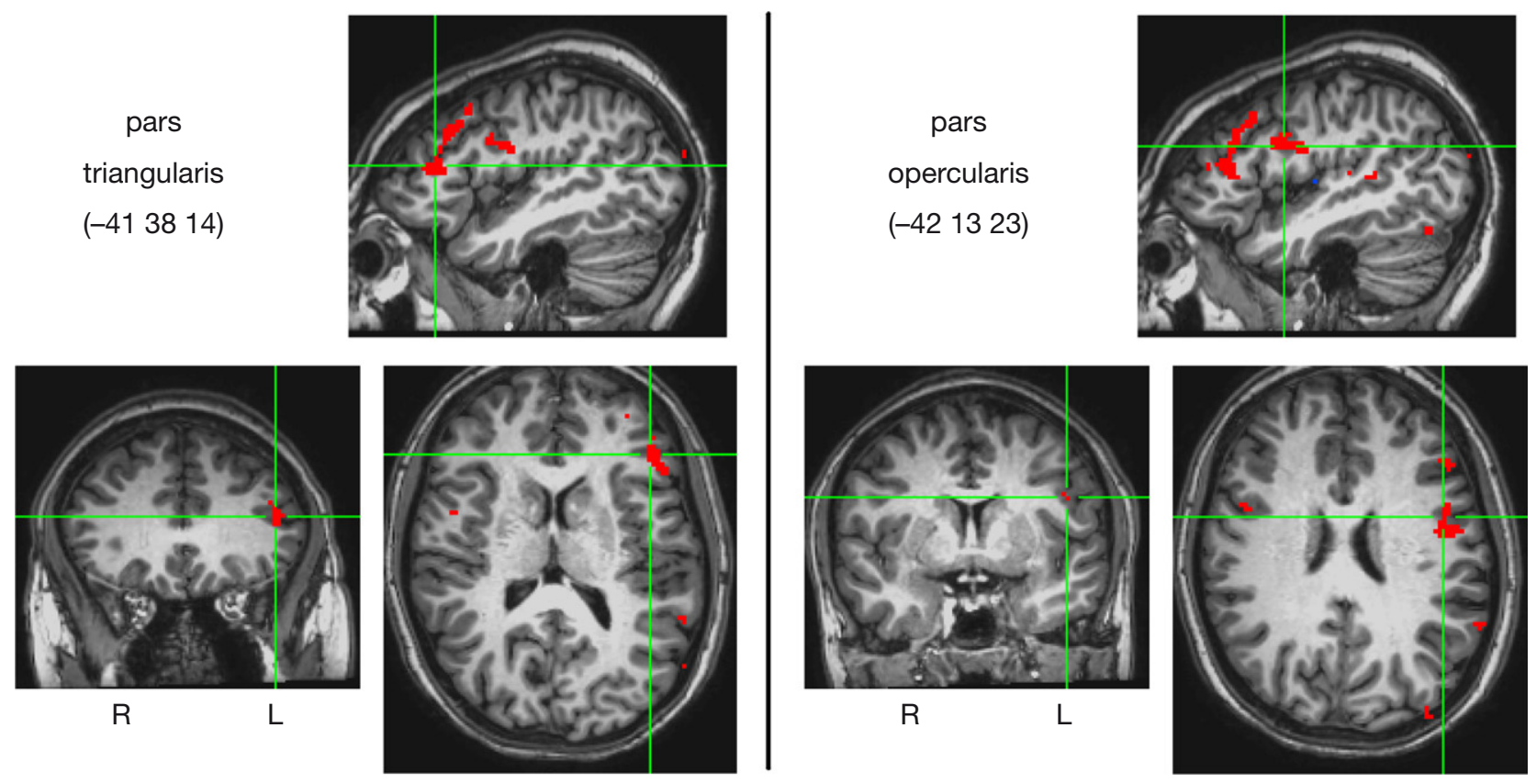

\section{B During Articulation:}
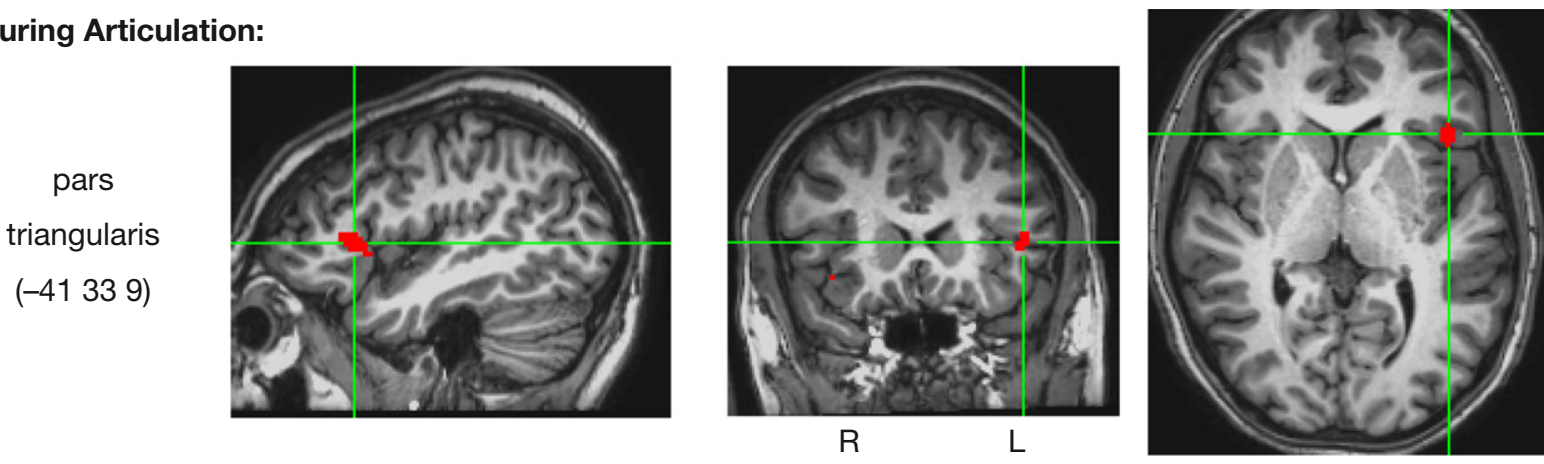

\section{During Finger-tapping Task:}
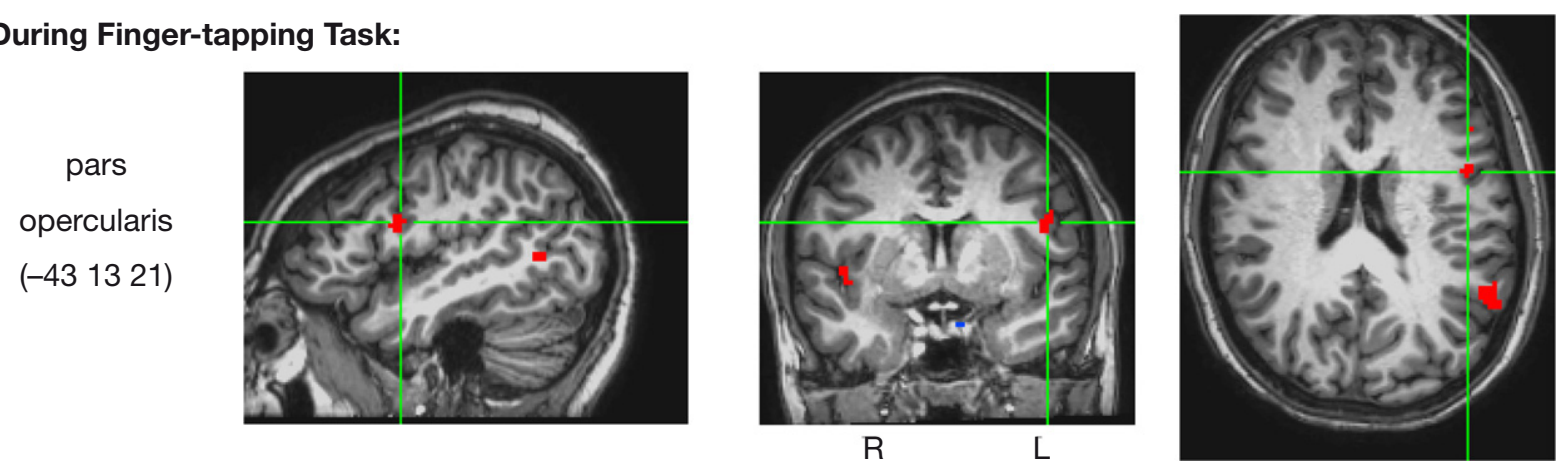

Figure 5 | Left inferior frontal voxel clusters more active during the presentation of object-relative clause sentences than subject-relative clause sentences $(\boldsymbol{p}<\mathbf{0 . 0 0 5})$. Clusters shown are those meeting these criteria while subjects were performing $(\boldsymbol{A})$ no secondary task, $(\boldsymbol{B})$ articulation, or $(\boldsymbol{C})$ a fingertapping task. Talairach coordinates for peak activity in each cluster are listed.

temporal gyrus, and a region in the vicinity of the left claustrum (Table 3).

During the finger-tapping task condition, much of the activation found in the no-secondary-task condition was again not evident, and again only a sub-region of Broca's area remained activated in the object-relative minus subject-relative contrast. This sub-region differed from that found in the speech articulation condition, however. During concurrent finger tapping, the object-relative minus subject-relative contrast yielded a focus of activation $(p<0.005)$ in the pars opercularis $(-431321)$

8 
Table 3 | Peak $\boldsymbol{t}$-values and talairach coordinates for these peaks (as well as approximate region based on these coordinates) for all voxel clusters that passed threshold $(p<0.005)$ for the OR versus SR sentence contrast in each condition, averaged across trials and subjects.

\begin{tabular}{|c|c|c|c|c|c|}
\hline \multirow[t]{2}{*}{ OR sentences $>$ SR sentences } & \multirow[t]{2}{*}{ Region } & \multicolumn{4}{|c|}{ Peak $t$-score in Cluster } \\
\hline & & $t$-Value & $x$ & $y$ & $z$ \\
\hline \multirow[t]{7}{*}{ With No Secondary Task } & L IFG & 6.82 & -41 & 38 & 14 \\
\hline & L IFG & 5.37 & -42 & 13 & 23 \\
\hline & L STG & 4.56 & -56 & -37 & 19 \\
\hline & LPCG & 5.21 & -54 & 4 & 31 \\
\hline & L MFG & 5.69 & -39 & 32 & 32 \\
\hline & L MFG & 5.22 & -32 & 56 & 19 \\
\hline & $\mathrm{R}$ IFG & 6.15 & 47 & 16 & 20 \\
\hline \multirow[t]{4}{*}{ During Articulation Task } & L IFG & 4.36 & -41 & 33 & 9 \\
\hline & L claustrum & 4.46 & -29 & 7 & -2 \\
\hline & R IFG & 4.23 & 37 & 27 & -9 \\
\hline & R STG & 3.89 & 46 & -32 & 12 \\
\hline \multirow[t]{6}{*}{ During Finger-Tapping Task } & L IFG & 4.1 & -43 & 13 & 21 \\
\hline & L IFG & 6.38 & -31 & 27 & -5 \\
\hline & L STG & 4.82 & -55 & -44 & 17 \\
\hline & L insula & 6.25 & -34 & 18 & 12 \\
\hline & L claustrum & 6.18 & -34 & -9 & 0 \\
\hline & R insula & 4.59 & 41 & 12 & -5 \\
\hline
\end{tabular}

IFG, Inferior frontal gyrus; STG, superior temporal gyrus; PCG, pre-central gyrus; MFG, middle frontal gyrus.

\section{Overlap of Regions Active During Articulation Alone \& To OR > SR During No Secondary Task}
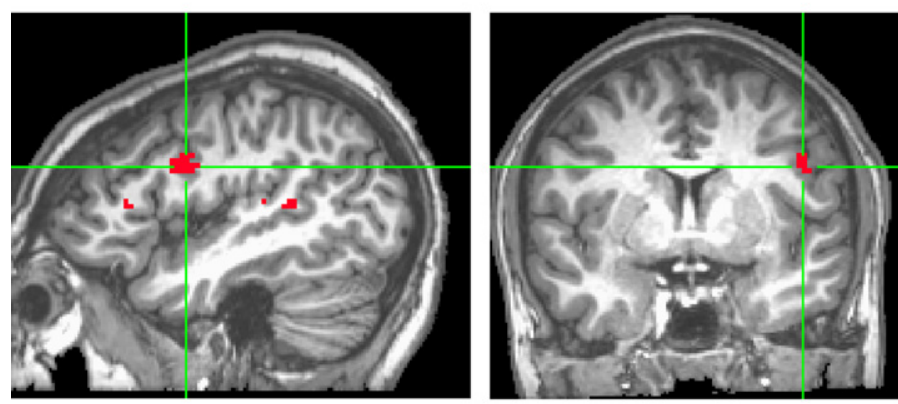

$\mathrm{R}$
L

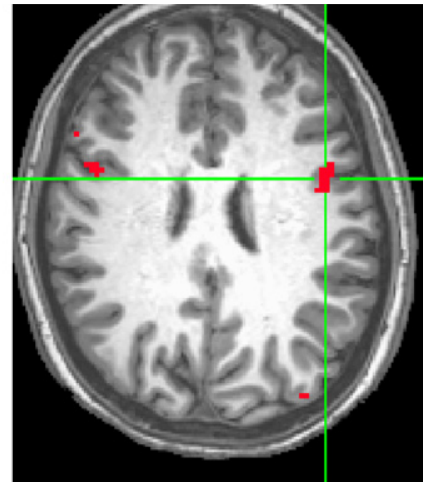

Figure 6 | Conjunction of regions active during articulation alone $(p<0.005)$ and regions more active during object-relative than subject-relative sentences during no secondary task $(\boldsymbol{p}<\mathbf{0 . 0 0 5})$. The crosshairs converge on the largest voxel meeting these criteria (left pars opercularis, -421225$)$.

Table 4 | Peak $\boldsymbol{t}$-test statistics/ $\boldsymbol{p}$-values for the OR versus SR sentence contrast in each condition for the clusters in Broca's area identified as more active for OR than SR sentences in the absence of a concurrent task.

OR $>$ SR Regions with no Secondary Task

\begin{tabular}{lll}
\cline { 2 - 3 } $\begin{array}{l}\text { OR sentences }> \\
\text { SR sentences }\end{array}$ & $\begin{array}{l}\text { L pars triangularis } \\
\mathbf{( - 4 1 ~ 3 8 ~ 1 4 )}\end{array}$ & $\begin{array}{l}\text { L pars opercularis } \\
\mathbf{( - 4 2 ~ 1 3 ~ 2 3 )}\end{array}$ \\
\hline With no secondary task & $6.82 / 0.0001$ & $5.37 / 0.0007$ \\
During articulation & $4.36 / 0.0009$ & $1.72 / 0.110$ \\
During finger-tapping task & $1.24 / 0.246$ & $4.10 / 0.002$
\end{tabular}

(Figure 5C, Table 3). This cluster's location and extent is almost identical to the left pars opercularis region found in the objectrelative vs subject-relative contrast during no secondary task. The left pars triangularis region that demonstrated an object-relative sentence preference in the absence of a secondary task did not approach threshold for the contrast during finger-tapping $(p>0.10)$ (Table 4). Other clusters passing threshold for the object-relative clause versus subject-relative clause sentence contrast during finger-tapping were found in the left superior temporal gyrus, the left claustrum, and bilaterally in the insula (Table 3).

Figure 7 shows mean peak amplitude plots for all conditions within the pars opercularis and pars orbitalis regions identified in the above analyses (i.e., the ROIs shown in Figure 5). Although the pars opercularis and pars triangularis regions identified by the OR > SR contrast during no concurrent task are not the exact same voxels identified by the contrasts during concurrent articulation and finger-tapping, respectively, comparing the mean peak amplitudes for the clusters (i.e. comparing the left and right sides of Figure 7) reveals that they have very similar 

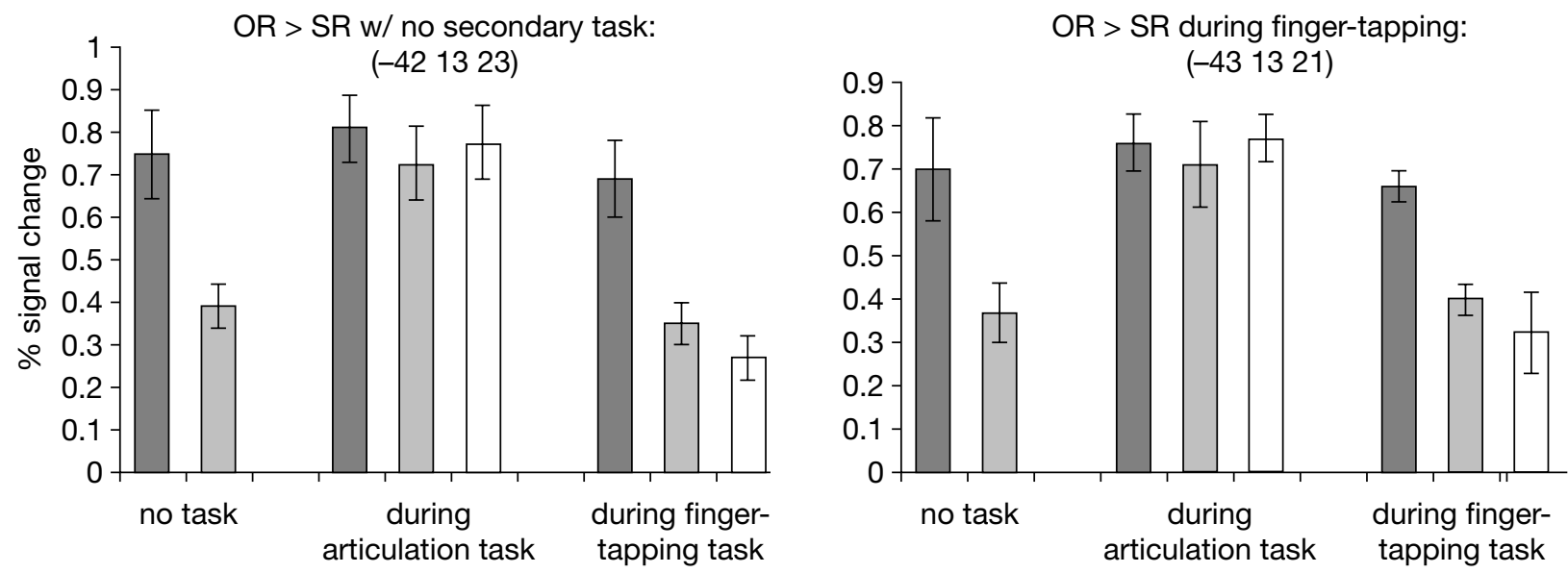

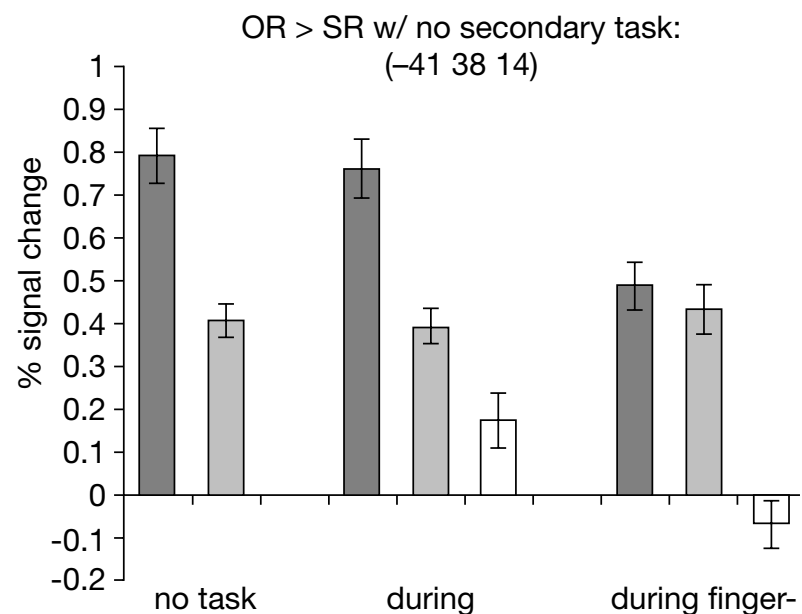
articulation task tapping task

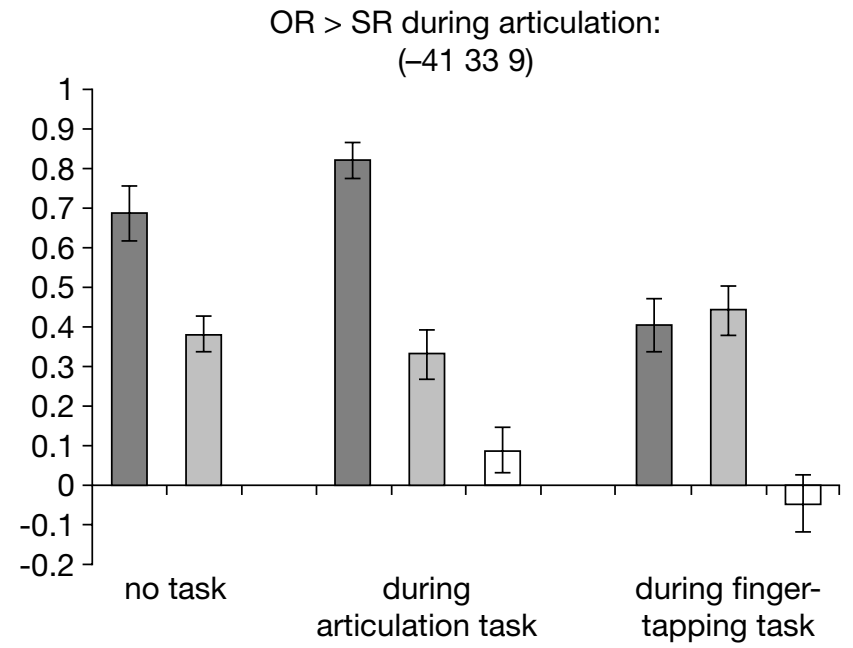

subject-relative articulation task

object-relative

Figure 7 | Mean peak amplitudes of the left pars opercularis $(A)$ and left pars triangularis $(B)$ regions more active during the perception of objectrelative sentences than during subject-relative sentences $(p<0.005)$ in the absence of a secondary task, or during one of the concurrent tasks, averaged across trials and subjects. Mean peak amplitudes of each cluster are shown for each sentence type presented during each task condition, as well as for just articulation and finger-tapping alone. Error bars represent $95 \%$ confidence intervals. The Talairach coordinates of each cluster's peak are given, and correspond to the contrast maps in Figure 5.

response properties. Considering the pars opercularis first, it is clear that speech articulation alone activates this region, and does so equally as well as the OR sentences with no concurrent task. Processing OR sentences during speech articulation does not increase activation levels over articulation or OR sentence comprehension alone. However, adding speech articulation to SR sentence comprehension does increase activation levels presumably reflecting a saturation of this region's activity due to the articulation task, as expected. Finally, speech articulation alone produces substantially more activation than performing the finger tapping task alone; finger tapping appears to have no effect on the activation levels in the pars opercularis associated with OR or SR sentence processing. In short, this pattern of results is consistent with the view that activation in this region is driven primarily by speech articulation: activation is maximal during articulation whether sentences are being processed or not (middle set of bars in Figure 7), or during the processing of high load sentences that draw on articulatory rehearsal (OR sentences in left and right sets of bars in Figure 7).

Although the pars opercularis and pars triangularis respond similarly during sentence comprehension in the absence of a secondary task, these two portions of Broca's area respond quite differently when a secondary task is introduced. Unlike the pars opercularis, in the pars triangularis, speech articulation alone produces little activation relative to both OR sentences and even SR sentences, and adding speech articulation to sentence processing does not modulate neural activity at all. Adding a sequential finger-tapping task to sentence processing does modulate neural activity in this region: despite the fact that the finger-tapping task alone yields no activation in the pars triangularis, adding 
the task nonetheless leads to a reduction in amplitude to the OR sentences such that there is no longer a difference between OR and SR sentences.

Thus, the secondary tasks both eliminate a (different) portion of the OR minus SR activation in Broca's area, but in different ways. Speech articulation eliminates the OR-SR activation in the pars opercularis by increasing activation in the SR sentence condition, presumably because articulation saturates activity, whereas finger tapping eliminates the OR-SR activation in the pars triangularis by decreasing activation in the OR sentence condition.

\section{DISCUSSION}

The present study investigated the nature of the contributions of Broca's area to sentence processing by comparing the effects of syntactic complexity under three conditions: (i) a baseline condition with no secondary task, (ii) during a concurrent speech articulation task, and (iii) during a concurrent fingertapping sequence task. Consistent with previous research (e.g. Caplan et al., 2000, 2008; Fiebach et al., 2005; Stromswold et al., 1996), in the baseline condition, we found a "sentence complexity effect" (greater activation for sentences with object relatives than subject relatives) in Broca's area (pars opercularis and pars triangularis). During the concurrent speech articulation condition, only a portion of the pars triangularis showed a sentence complexity effect suggesting that the complexity effect found in other regions, the pars opercularis in particular, is attributable to the articulatory rehearsal mechanism of verbal working memory. Specifically, this finding is consistent with the following claims: Under high processing load conditions, such as sentences with object-extracted relative clauses, verbal working memory can be recruited to assist comprehension. This is reflected by greater brain activity in regions supporting verbal working memory (such as the pars opercularis) under high load conditions. When the contributions of verbal working memory are reduced via articulatory suppression, comprehension performance declines (Experiment 1 and behavioral data from Experiment 2), and brain regions supporting articulatory rehearsal (pars opercularis) are no longer modulated by sentence complexity because activity in these regions is saturated by the secondary articulatory task (fMRI data from Experiment 2). Put differently, this interpretation holds that the articulatory rehearsal mechanism is driving the sentence complexity effect in the pars opercularis because (i) complex sentences recruits verbal working memory more than less complex sentences, and (ii) the pars opercularis supports the articulatory rehearsal component of verbal working memory. By adding a secondary speech articulation task, which should maximally drive articulatory rehearsal and therefore the pars opercularis, the sentence complexity effect should be eliminated via a raising of activity levels during the simpler sentences to equal that of the complex sentences. This is precisely the pattern we observed. Furthermore, notice that adding a secondary task to the comprehension of complex sentences did not produce an increase in pars opercularis activity levels. This makes sense if processing complex sentences and performing articulatory rehearsal are tapping into the same pars opercularis resources.

The claim that the pars opercularis supports a verbal working memory contribution to sentence processing further predicts that the pars opercularis should show a sentence complexity effect during a secondary task that does not involve verbal working memory, even if such a task results in a comprehension performance decrement. This is precisely what we found in the secondary finger-tapping task: performance declined during finger-tapping relative to no-secondary-task (Experiment 1, and marginally significant behavioral effect in Experiment 2), yet the pars opercularis still showed a sentence complexity effect. We therefore suggest that the pars opercularis contributes to sentence comprehension via its role in the articulatory rehearsal component of verbal working memory. This contrasts with Fiebach et al.'s (2005) position that the pars opercularis' response to syntactic complexity is because this area is critical for syntactic-specific working memory (i.e., a mechanism for holding syntactic information while new syntactic structures are being processed and integrated with the previous information).

Of the regions that showed a sentence complexity effect during the no-secondary-task condition, one area, a focus in the pars triangularis, continued to show a complexity effect even during the secondary speech articulation task. This is consistent with the findings of Caplan et al. (2000) who report a sentence complexity effect in a similar region during articulatory suppression. It is unclear what functional role this region may be playing in sentence comprehension. Because the complexity effect found in the pars triangularis was not evident in our study during concurrent finger tapping, one might be tempted to suggest that the region supports some form of domain general sequence processing that might be useful for syntactic operations. According to such a view, under conditions of concurrent finger tapping this resource would be less available for sentence comprehension. This would be broadly consistent with recent imaging studies that have found that inferior frontal regions, including the pars triangularis, demonstrate an increase in activity when subjects observe or imagine performing goal-directed movement sequences, detect musical sequences, or process sequences of events (Koelsch et al., 2002; Schubotz and von Cramon, 2002, 2004). In addition, patients with lesions to this region have significant deficits in sequence prediction tasks compared to identification tasks, and compared to patients with prefrontal or parietal lesions (Schubotz et al., 2004). However, the finger-tapping task alone did not produce activation in the pars triangularis, nor did it yield an increase in activation in that region when performed concurrently with sentence comprehension (Figures 4 and 5). For this reason, it seems unlikely that the pars triangularis contributes to sentence comprehension via a sequence processing mechanism. Further research is clearly needed to understanding the functional role of the pars triangularis in sentence processing.

Caplan et al. (2008) suggest that the left IFG, generally, supports sentence processing via two possible functions: rehearsal and thematic role checking (i.e. evaluating various thematic role assignment possibilities). These authors further point out that evaluating various thematic role possibilities is similar to another proposed function of the inferior frontal gyrus, namely selecting a response from a set of alternatives (Kan and ThompsonSchill, 2004; Novick et al., 2005). Our findings support the claim of a rehearsal function, and specify that the pars opercularis is particularly involved in this respect. It is possible that our pars triangularis location contributes to sentence processing via operations involved in response selection, although it is not clear why the performance of a finger-tapping task (but not a speech articulation task) should decrease activity for more complex sentences in this area.

\section{CONCLUSION}

Findings from our first experiment indicate that the articulatory rehearsal component of verbal working memory contributes to 
sentence comprehension. In particular, this mechanism appears to facilitate comprehension of high-load sentences, such as those containing semantically reversible object-extracted relative clauses. Our fMRI results identified the pars opercularis portion of Broca's area as an important region in this respect. The pars triangularis portion of Broca's area also may contribute to sentence comprehension, but in a different manner. The nature of the pars triangularis' contribution to sentence comprehension requires further study, but one possibility is that it plays a role in selecting from various thematic role relation possibilities associated within a sentence (Caplan et al., 2008).

\section{CONFLICT OF INTEREST STATEMENT}

The authors declare that the research was conducted in the absence of any commercial or financial relationships that could be construed as a potential conflict of interest.

\section{ACKNOWLEDGMENTS}

This research was funded by G.Hickok's NIH RO1 grant DC03681. Please send correspondence to Gregory Hickok, Department of Cognitive Sciences, University of California, Irvine, Irvine, CA 92697.

\section{REFERENCES}

Baddeley, A. D. (1981). The role of subvocalisation in reading. Q. J. Exp. Psychol. $33 \mathrm{~A}, 439-454$.

Baddeley, A. D. (1986). Working Memory. Oxford, Clarendon Press.

Baddeley, A. D., and Hitch, G. J. (1974). Working memory. In The Psychology of Learning and Motivation, Vol. 8, Advances in Research and Theory, G. A. Bower ed. (New York, Academic Press), pp. 47-89.

Baddeley, A. D., Thomson, N., and Buchanan, M. (1975). Word length and the structure of short-term memory. J. Verbal Learn. Verbal Behav. 14, 575-589.

Berndt, R. S., Mitchum, C. C., and Haendiges, A. N. (1996). Comprehension of reversible sentences in 'agrammatism': a meta-analysis. Cognition 58, 289-308.

Besner, D. V. M., and Davelaar, E. (1982). Basic processes in reading: two phonological codes. Can. J. Psychol. 36, 701-711.

Boyle, R., and Coltheart, V. (1996). Effects of irrelevant sounds on phonological coding in reading comprehension and short-term memory. Q. J. Exp. Psychol. 49A, 398-416.

Bradley, D. C., Garrett, M. E., and Zurif, E. B. (1980). Syntactic deficits in Broca's aphasia. In Biological Studies of Mental Processes, D. Caplan ed. (Cambridge, MA, MIT Press).

Buchsbaum, B., Hickok, G., and Humphries, C. (2001). Role of left posterior superior temporal gyrus in phonological processing for speech perception and production. Cogn. Sci. 25, 663-678.

Caplan, D., Alpert, N., and Waters, G. (1998). Effects of syntactic structure and propositional number on patterns of regional cerebral blood flow. J. Cogn. Neurosci. 10, 541-552.

Caplan, D., Alpert, N., and Waters, G. (1999). PET studies of syntactic comprehension with auditory sentence presentation. NeuroImage 9, 343-351.

Caplan, D., Alpert, N., Waters, G., and Olivier, A. (2000). Activation of Broca's area by syntactic processing under conditions of concurrent articulation. Hum. Brain Mapp. 9, 65-71.

Caplan, D., and Hildebrandt, N. (1988). Disorders of Syntactic Comprehension. Cambridge, MA, MIT Press.

Caplan, D., Stanczak, L., and Waters, G. (2008). Syntactic and thematic constrain effects on blood oxygenation level dependent signal correlates of comprehension of relative clauses. J. Cogn. Neurosci. 20, 643-656.

Caplan, D., and Waters, G. (1999). Verbal working memory and sentence comprehension. Behav. Brain Sci. 22, 77-126.

Caramazza, A., and Zurif, E. B. (1976). Dissociation of algorithmic and heuristic processes in language comprehension: evidence from aphasia. Brain Lang. 3 $572-582$.

Coltheart, V. (1993). Effects of phonological similarity and concurrent irrelevant articulation on short-term-memory recall of repeated and novel word lists Mem. Cogn. 21, 539-545.

Cox, R. W., and Hyde, J. S. (1997). Software tools for analysis and visualization of fMRI data. NMR Biomed. 10, 171-178.

Cox, R. W., and Jesmanowicz, A. (1999). Real-time 3d image registration for functional MRI. Magn. Reson. Med. 42, 1014-1018.
Daneman, M., and Carpenter, P. A. (1980). Individual differences in working memory and reading. J. Verbal Learn. Verbal Behav. 19, 450-466.

Dapretto, M., and Bookheimer, S. Y. (1999). Form and content: dissociating syntax and semantics in sentence comprehension. Neuron 24, 427-432.

De Beni, R., Pazzaglia, F., Gyselinck, V., and Meneghetti, C. (2005). Visuospatial working memory and mental representation of spatial descriptions. Eur. J. Cognit. Psychol. 17, 77-95.

De Renzi, E., and Nichelli, P. (1975). Verbal and nonverbal short-term memory impairment following hemispheric damage. Cortex 11, 341-351.

Dronkers, N. F., Shapiro, J. K., Redfern, B., and Knight, R. T. (1992). The role of Broca's area in Broca's aphasia. J. Clin. Exp. Neuropsychol. 14, 52-53.

Dronkers, N. F., Wilkins, D. P., Van Valin, R. Jr, Redfern, B., and Jaeger, J. (2004). Lesion analysis of the brain areas involved in language comprehension. Cognition 92, 145-177.

Fiebach, C. J., Schlesewsky, M., Lohmann, G., von Cramon, D. Y., and Friederici, A. D. (2005). Revisiting the role of Broca's area in sentence processing: syntactic integration versus syntactic working memory. Hum. Brain Mapp. 24, 79-91.

Gleason, J. B., Goodglass, H., Green, E., Ackerman, N., and Hyde, M. (1975). The retrieval of syntax in Broca's aphasia. Brain Lang. 2, 451-471.

Goodglass, H. (1968). Studies in the grammar of aphasics. In Developments in Applied Psycholinguistic Research, S. Rosenberg and J. Koplin, eds (New York, MacMillan).

Goodglass, H. (1976). Agrammatism. In Studies in Neurolinguistics Vol. 1., H. Whitaker and H. A. Whitaker eds (New York, Academic Press).

Goodglass, H., and Berko, J. (1960). Aphasia and inflectional morphology in English. J. Speech Hear. Res. 3, 257-267.

Goodglass, H., and Kaplan, E. (1972). The Assessment of Aphasia and Related Disorders. Philadelphia, Lea \& Febiger.

Grier, J. B. (1971). Nonparametric indexes for sensitivity and bias: Computing formulas. Psychol. Bull. 75, 424-429.

Grodzinsky, Y. (1984). The syntactic characterization of agrammatism. Cognition $16,99-120$

Grodzinsky, Y. (2000). The neurology of syntax: language use without Broca's area. Behav. Brain Sci. 23, 1-71.

Hanten, G., and Martin, R. C. (2000). Contributions of phonological and semantic short-term memory to sentence processing: evidence from two cases of closed head injury in children. J. Mem. Lang. 43, 335-361.

Hickok, G., Buchsbaum, B., Humphries, C., and Muftuler, T. (2003). Auditorymotor interaction revealed by fMRI: speech, music, and working memory in area Spt. J. Cogn. Neurosci. 15, 673-682.

Humphries, C., Buchsbaum, B., and Hickok, G. (2001). Role of anterior temporal cortex in auditory sentence comprehension: an fMRI study. Neuroreport 12 , $1749-1752$.

Humphries, C., Love, T., Swinney, D., and Hickok, G. (2005). Response of anterior temporal cortex to syntactic and prosodic manipulations during sentence processing. Hum. Brain Mapp. 26, 128-138.

Jonides, J., Schumacher, E. H., Smith, E. E., Lauber, E. J., Awh, E., Satosy, M., and Koeppe, R. A. (1997). Verbal working memory load effects regional brain activation as measured by PET. J. Cogn. Neurosci. 9, 462-475.

Just, M. A., and Carpenter, P. A. (1992). A capacity theory of comprehension: individual differences in working memory. Psychol. Rev. 99, 122-149.

Just, M. A., Carpenter, P. A., Keller, T. A., Eddy, W. F., and Thulborn, K. R. (1996). Brain activation modulated by sentence comprehension. Science 274, 114-116.

Kan, I. P., and Thompson-Schill, S. L. (2004). Selection from perceptual and conceptual representations. Cogn. Affect. Behav. Neurosci. 4, 466-482.

Kean, M. L. (1977). The linguistic interpretation of aphasic syndromes: agrammatism in Broca's aphasia, an example. Cognition 5, 9-45.

Kean, M. L. (1995). The elusive character of agrammatism. Brain Lang. 50, 369-384.

Koelsch, S., Gunter, T. C., von Cramon, D. Y., Zysset, S., Lohmann, G., and Friederici, A. D. (2002). Bach speaks: a cortical 'language network' serves the processing of music. NeuroImage 17, 956-966.

Linebarger, M. C. (1990). Neuropsychology of sentence parsing. In Cognitive Neuropsychology and Neurolingustics: Advances in Models of Cognitive Function and Impairment, A. Caramazza ed. (Hillsdale, NJ, Erlbaum), pp. 55-122.

Linebarger, M. C., Schwartz, M. F., and Saffran, E. M. (1983). Sensitivity to grammatical structure in so-called agrammatic aphasics. Cognition 13, 361-392.

Love, T., and Oster, E. (2002). On the categorization of aphasic typologies: the SOAP (a test of syntactic complexity). J. Psycholinguist. Res. 31, 503-529.

Martin, R. C. (2003). Language processing: functional organization and neuroanatomical basis. Ann. Rev. Psychol. 54, 55-89.

Mazoyer, B. M., Tzourio, N., Frak, V., Syrota, A. Murayama, N., et al. (1993). The cortical representation of speech. J. Cogn. Neurosci. 5, 467-479.

Mohr, J. P. (1976). Broca's area and Broca's aphasia. In Studies in neurolinguistics, Vol. 1, H. Whitaker and H. A. Whitaker eds (New York, Academic Press).

Mohr, J. P., Pessin, M. S., Finkelstein, S., Funkenstein, H. H., Duncan, G. W., and Davis, K. R. (1978). Broca's aphasia: pathological and clinical. Neurology 28, 311-324. 
Novick, J. M., Trueswell, J. C., and Thompson-Schill, S. L. (2005). Cognitive control and parsing: reexamining the role of Broca's area in sentence comprehension. Cogn. Affect. Behav. Neurosci. 5, 263-281.

Postle, B. R., Berger, J. S., Goldstein, J. H., Curtis, C. E., and D'Esposito, M. (2001) Behavioral and neurophysiological correlates of episodic coding, proactive interference, and list length effects in a running span verbal working memory task. Cogn. Affect. Behav. Neurosci. 1, 10-21.

Rogalsky, C., and Hickok, G. (2008). Selective attention to semantic and syntactic features modulates sentence processing networks in anterior temporal cortex. Cereb. Cortex, advanced access online publication on July 31, 2008.

Rorden, C., and Brett, M. (2000). Stereotactic display of brain lesions. Behav Neurol. 12, 191-200.

Schubotz, R. I., Sakreida, K., Tittgemeyer, M., and von Cramon, D. Y. (2004). Motor areas beyond motor performance: deficits in serial prediction following ventrolateral premotor lesions. Neuropsychology 18, 638-645.

Schubotz, R. I., and von Cramon, D. Y. (2002). Predicting perceptual events activates corresponding motor schemes in lateral premotor cortex: an fMRI study. NeuroImage 15, 787-796.

Schubotz, R. I., and von Cramon, D. Y. (2004). Sequences of abstract nonbiological stimuli share ventral premotor cortex with action observation and imagery. J. Neurosci. 24, 5467-5474.

Slowiaczek, M. L., and Clifton, C. (1980). Subvocalization and reading for meaning. J. Verbal Learn. Verbal Behav. 19, 573-582.
Smith, E. E., and Jonides, J. (1997). Working memory: a view from neuroimaging. Cogn. Psychol. 33, 5-42.

Smith, E. E., Jonides, J., Marshuetz, C., and Koeppe, R. A. (1998). Components of verbal working memory: evidence from neuroimaging. Proc. Natl. Acad. Sci. U.S.A. 95, 876-882.

Stromswold, K., Caplan, D., Alpert, N., and Rauch, S. (1996). Localization of syntactic comprehension by positron emission tomography. Brain Lang. 52, 452-473.

Swinney, D., and Taylor, O. (1971). Short-term memory recognition search in aphasics. J. Speech Hear. Res. 14, 578-588.

Takahashi, M. (2007). Cognitive processes in sentence comprehension during silent and oral reading: role of attentional resources and phonological encoding. Jpn. J. Educ. Psychol. 55, 538-549.

Talairach, J., and Tournoux, P. (1988). Co-planar Stereotaxic Altas of the Human Brain. New York, Thieme Medical Publishers, p. 122.

Vandenberge, R., Nobre, A. C., and Price, C. J. (2002). The response of left temporal cortex to sentences. J. Cogn. Neurosci. 14, 550-560.

Waters, G., Caplan, D., and Hildebrandt, N. (1991). On the structure of verbal short-term memory and its functional role in sentence comprehension: evidence from neuropsychology. Cogn. Neuropsychol. 8, 81-126.

Wulfeck, B. (1988). Grammaticality judgments and sentence comprehension in agrammatic aphasia. J. Speech Hear. Disord. 31, 72-81.

Zatorre, R. J., Evans, A. C., Meyer, E., and Gjedde, A. (1992). Lateralization of phonetic and pitch discrimination in speech processing. Science 256, 846-849. 